\title{
Nullcone Fronts of Spacelike Framed Curves in Minkowski 3-Space
}

\author{
Pengcheng Li (D) and Donghe Pei * (D) \\ School of Mathematics and Statistics, Northeast Normal University, Changchun 130024, China; \\ lipc732@nenu.edu.cn \\ * Correspondence: peidh340@nenu.edu.cn
}

check for

updates

Citation: Li, P.; Pei, D. Nullcone Fronts of Spacelike Framed Curves in Minkowski 3-Space. Mathematics 2021, 9, 2939. https://doi.org/10.3390/ math9222939

Academic Editor: Josef Mikeš

Received: 19 October 2021

Accepted: 16 November 2021

Published: 18 November 2021

Publisher's Note: MDPI stays neutral with regard to jurisdictional claims in published maps and institutional affiliations.

Copyright: (c) 2021 by the authors. Licensee MDPI, Basel, Switzerland. This article is an open access article distributed under the terms and conditions of the Creative Commons Attribution (CC BY) license (https:// creativecommons.org/licenses/by/ $4.0 /)$.

\begin{abstract}
The investigation of objects in Minkowski space is of great significance, especially for those objects with mathematical and physical backgrounds. In this paper, we study nullcone fronts, which are formed by the light rays emitted from points on a spacelike curve. However, if the spacelike curve is singular, then we cannot use the usual tools and methods to study related issues. To solve these problems, we show the definition of spacelike framed curves in Minkowski 3-space, whose original curves may contain singularities. Then, the singularities of the nullcone fronts are characterized by using framed curvatures of spacelike framed curves. Finally, we exhibit some examples to illustrate our results.
\end{abstract}

Keywords: nullcone fronts; spacelike framed curves; Minkowski 3-space; singularities

\section{Introduction}

The pseudo-Riemannian space has a vital position in the research of differential geometry and physics, among which the Minkowski space is an important space model in relativity [1]. Curves and their related submanifolds are attractive research subjects in differential geometry and physics [2-6] particularly for geometric objects that have interesting optical backgrounds and meanings. For example, F. Ateş et al. studied special curves formed by reflected light rays in different spaces and analyzed the singular properties of related submanifolds $[7,8]$. Moreover, the nullcone front in this paper can be regarded as a surface formed by the trajectory of light rays emitted from each point on a spacelike curve. This is a special relativity understanding of Fermat's principle that regards the light as particles [9].

If a spacelike curve is regular, we can use the Frenet frame to obtain most of the information of the curve, and then we can characterize properties of the curve and its related submanifolds [10]. However, as we know, for singular curves, the Frenet frame does not exist at the singularities, such as cusps, inflection points, etc., which makes it impossible to utilize the Frenet frame to study the curve and its related submanifolds near the singularities.

For this reason, some authors constructed or adopted different tools to study the objects in different spaces [11-13]. Particularly, S. Honda and M. Takahashi defined framed curves in Euclidean space. Then, they used framed curves to character the singular curve in Euclidean 3-space. This is not only the promotion of Frenet curves but also the promotion of Legendre curves [14]. Subsequently, using the framed curve as a tool, some authors have studied geometric objects in different spaces [15-17].

The spacelike curve is a classical geometric object of semi-Euclidean space. For instance, S. Izumiya, the second author and T. Sano studied regular spacelike curves in Minkowski 3-space [18]. However, spacelike curves may contain singularities. To solve this kind of problem, in present paper, we extend the notion of framed curve to the Minkowski 3-space. We show the definition of spacelike framed curves in Minkowski 3-space. Then, 
the nullcone fronts of a spacelike framed curve are defined. Then, the singularities of them are classified as a generalization of the conclusions of S. Izumiya et al [18].

The brief structure of the present paper is as follows. In the next section, we introduce the fundamental definitions in Minkowski 3-space. We show the definition of spacelike framed curves. Then, Frenet type formulae of a spacelike framed curve are given. In Section 3, we show the definitions of nullcone fronts and give the main classification theorem of this paper (Theorem 3). In Section 4, we review the criteria for singularities of fronts. We show the proof of Theorem 3. Finally, we show several (singular) curves as examples in Section 5, whose nullcone fronts may have cuspidal edges or swallowtails.

\section{Preliminaries}

Let $\mathbb{R}^{3}$ be the 3-dimensional real vector space. The pseudo scalar product of $x$ and $y$ is defined by

$$
\langle\boldsymbol{x}, \boldsymbol{y}\rangle=-x_{1} y_{1}+x_{2} y_{2}+x_{3} y_{3}
$$

for any vectors $x=\left(x_{1}, x_{2}, x_{3}\right), y=\left(y_{1}, y_{2}, y_{3}\right)$ in $\mathbb{R}^{3}$. Then, the space $\mathbb{R}_{1}^{3}=\left(\mathbb{R}^{3},\langle\cdot, \cdot\rangle\right)$ is called the pseudo-Riemannian 3-space with index 1 or the Minkowski 3-space.

If a non-zero vector $x \in \mathbb{R}_{1}^{3}$ satisfies $\langle\boldsymbol{x}, \boldsymbol{x}\rangle>0,\langle\boldsymbol{x}, \boldsymbol{x}\rangle<0$ or $\langle\boldsymbol{x}, \boldsymbol{x}\rangle=0$, then the non-zero vector $x$ is called a spacelike, timelike or null vector respectively. Let $\gamma: I \subset \mathbb{R} \rightarrow \mathbb{R}_{1}^{3}$; $t \mapsto \gamma(t)$ be a regular curve, that is, $\gamma^{\prime}(t) \neq \mathbf{0}$ for any $t \in I$. We say that a curve $\gamma(t)$ is a spacelike, timelike or null curve if its tangent vector $\gamma^{\prime}(t)$ is spacelike, timelike or null for any $t \in I$. The category to which a given vector or curve belongs is called its causal character.

Three kinds of pseudo-spheres in $\mathbb{R}_{1}^{3}$ are defined as follows.

The pseudo 2-sphere is defined by

$$
\mathbb{S}_{1}^{2}=\left\{x \in \mathbb{R}_{1}^{3} \mid\langle x, x\rangle=1\right\},
$$

the hyperbolic 2-space is defined by

$$
\mathbb{H}_{0}^{2}=\left\{x \in \mathbb{R}_{1}^{3} \mid\langle x, x\rangle=-1\right\},
$$

and the nullcone with the vertex $p$ is defined by

$$
\mathbb{N C}_{p}=\left\{x \in \mathbb{R}_{1}^{3} \mid\langle\boldsymbol{x}-\boldsymbol{p}, \boldsymbol{x}-\boldsymbol{p}\rangle=0\right\} .
$$

Moreover, for any vectors $x$ and $y$ in $\mathbb{R}_{1}^{3}$ with components as above, the pseudo vector product of them is given by

$$
\boldsymbol{x} \wedge \boldsymbol{y}=\left|\begin{array}{rrr}
-\boldsymbol{e}_{1} & \boldsymbol{e}_{2} & \boldsymbol{e}_{3} \\
x_{1} & x_{2} & x_{3} \\
y_{1} & y_{2} & y_{3}
\end{array}\right|,
$$

where $\left\{\boldsymbol{e}_{1}, \boldsymbol{e}_{2}, \boldsymbol{e}_{3}\right\}$ is the canonical basis of $\mathbb{R}_{1}^{3}$.

On the other hand, a spacelike framed curve in Minkowski space is a spacelike curve with a set of moving frame fields along the curve.

Let $\gamma: I \rightarrow \mathbb{R}_{1}^{3}$ be a spacelike curve. Then, the map $\left(\gamma, v_{1}, v_{2}\right): I \rightarrow \mathbb{R}_{1}^{3} \times \Delta$ is called a spacelike framed curve if

$$
\left\langle\gamma^{\prime}(t), v_{1}(t)\right\rangle=0, \quad\left\langle\gamma^{\prime}(t), v_{2}(t)\right\rangle=0, \quad \forall t \in I,
$$

where

$$
\Delta=\left\{\left(\boldsymbol{v}_{1}, \boldsymbol{v}_{2}\right) \in \mathbb{S}_{1}^{2} \times \mathbb{H}_{0}^{2} \mid\left\langle\boldsymbol{v}_{1}(t), \boldsymbol{v}_{2}(t)\right\rangle=0\right\}
$$

or

$$
\Delta=\left\{\left(\boldsymbol{v}_{1}, \boldsymbol{v}_{2}\right) \in \mathbb{H}_{0}^{2} \times \mathbb{S}_{1}^{2} \mid\left\langle\boldsymbol{v}_{1}(t), \boldsymbol{v}_{2}(t)\right\rangle=0\right\} .
$$

At this point, the curve $\gamma(t)$ is called the original curve of the spacelike framed curve. Then, we define $\mu(t)=v_{1}(t) \wedge v_{2}(t)$, which is a spacelike vector field. Moreover, we can 
find a smooth function $\alpha(t)$ that satisfies $\gamma^{\prime}(t)=\alpha(t) \boldsymbol{\mu}(t)$. It is easy to perceive that the original curve $\gamma(t)$ is singular at the point $t_{0}$ if and only if $\alpha\left(t_{0}\right)=0$.

Remark 1. In accordance with the causal character of the component of the frame field, we know that there are three kinds of frame fields for a spacelike curve [19]. In consideration of the objects we studied in the present paper, only two are given here.

We denote $\delta(t)=\operatorname{sign}\left(\boldsymbol{v}_{1}(t)\right)=\left\langle\boldsymbol{v}_{1}(t), \boldsymbol{v}_{1}(t)\right\rangle$, and then the Frenet type formulae for a spacelike framed curve are given as follows.

$$
\left(\begin{array}{c}
\boldsymbol{v}_{1}^{\prime}(t) \\
\boldsymbol{v}_{2}^{\prime}(t) \\
\boldsymbol{\mu}^{\prime}(t)
\end{array}\right)=\left(\begin{array}{ccc}
0 & \ell_{1}(t) & \ell_{2}(t) \\
\ell_{1}(t) & 0 & \ell_{3}(t) \\
-\delta(t) \ell_{2}(t) & \delta(t) \ell_{3}(t) & 0
\end{array}\right)\left(\begin{array}{c}
\boldsymbol{v}_{1}(t) \\
\boldsymbol{v}_{2}(t) \\
\boldsymbol{\mu}(t)
\end{array}\right)
$$

where $\ell_{1}(t)=\left\langle v_{1}^{\prime}(t), v_{2}(t)\right\rangle, \ell_{2}(t)=\left\langle v_{1}^{\prime}(t), \mu(t)\right\rangle, \ell_{3}(t)=\left\langle v_{2}^{\prime}(t), \mu(t)\right\rangle$. We call functions $\left(\alpha(t), \ell_{1}(t), \ell_{2}(t), \ell_{3}(t)\right)$ the curvature of the spacelike framed curve.

The existence and uniqueness theorems for spacelike framed curves are shown as follows.

Theorem 1 (The Existence Theorem). Let $\left(\alpha, \ell_{1}, \ell_{2}, \ell_{3}\right): I \rightarrow \mathbb{R}^{4}$ be a smooth map. There exists a spacelike framed curve $\left(\gamma, v_{1}, v_{2}\right): I \rightarrow \mathbb{R}_{1}^{3} \times \Delta$ whose curvature of the spacelike framed curve is $\left(\alpha, \ell_{1}, \ell_{2}, \ell_{3}\right)$.

Proof. Let $\theta, \omega: I \rightarrow \mathbb{R}$ be any functions with the properties that $\theta^{\prime}(t) \tan \omega(t)=\ell_{1}(t)$, $\omega^{\prime}(t) \sec \omega(t)=\ell_{2}(t),-\theta^{\prime}(t) \sec \omega(t)=\ell_{3}(t)$ for all $t \in I$. Moreover, let

$$
\begin{aligned}
\boldsymbol{v}_{1}(t) & =(-\sec \omega(t), \tan \omega(t) \cos \theta(t), \tan \omega(t) \sin \theta(t)), \\
\boldsymbol{v}_{2}(t) & =(0,-\sin \theta(t), \cos \theta(t)), \\
\boldsymbol{\mu}(t) & =(-\tan \omega(t), \sec \omega(t) \cos \theta(t), \sec \omega(t) \sin \theta(t))
\end{aligned}
$$

be the vector fields in $\mathbb{H}_{0}^{2}$ and $\mathbb{S}_{1}^{2}$. We define smooth functions $x(t), y(t), z(t)$ that satisfy $x^{\prime}(t)=-\alpha(t) \tan \omega(t), y^{\prime}(t)=\alpha(t) \sec \omega(t) \cos \theta(t)$, and $z^{\prime}(t)=\alpha(t) \sec \omega(t) \sin \theta(t)$. Then, $\gamma: I \rightarrow \mathbb{R}_{1}^{3}$ is defined by $\gamma(t)=(x(t), y(t), z(t))$, that is,

$$
\gamma(t)=\left(-\int \alpha(t) \tan \omega(t) \mathrm{d} t, \int \alpha(t) \sec \omega(t) \cos \theta(t) \mathrm{d} t, \quad \int \alpha(t) \sec \omega(t) \sin \theta(t) \mathrm{d} t\right)
$$

It follows that $\gamma^{\prime}(t)=\alpha(t) \mu(t), v_{1}^{\prime}(t)=\ell_{1}(t) v_{2}(t)+\ell_{2}(t) \mu(t), v_{2}^{\prime}(t)=\ell_{1}(t) v_{1}(t)+$ $\ell_{3}(t) \mu(t)$ and $\left\langle\gamma^{\prime}(t), \boldsymbol{v}_{i}(t)\right\rangle=0, i=1,2$ for all $t \in I$. Therefore, there exists a spacelike framed curve $\left(\gamma, v_{1}, v_{2}\right): I \rightarrow \mathbb{R}_{1}^{3} \times \Delta$ whose curvature of the spacelike framed curve is $\left(\alpha, \ell_{1}, \ell_{2}, \ell_{3}\right)$. Similarly, we can obtain the existence theorem for the case of $v_{1} \in \mathbb{S}_{1}^{2}$ and $v_{2} \in \mathbb{H}_{0}^{2}$.

Definition 1. Let $\left(\gamma, \boldsymbol{v}_{1}, \boldsymbol{v}_{2}\right)$ and $\left(\tilde{\gamma}, \tilde{\boldsymbol{v}}_{1}, \tilde{\boldsymbol{v}}_{2}\right): I \rightarrow \mathbb{R}_{1}^{3} \times \Delta$ be spacelike framed curves. We say that $\left(\gamma, v_{1}, v_{2}\right)$ and $\left(\tilde{\gamma}, \tilde{v}_{1}, \tilde{v}_{2}\right)$ are congruent as spacelike framed curves through a Lorentzian motion if there exist a matrix $M$ and a constant vector $c \in \mathbb{R}_{1}^{3}$ such that

$$
\tilde{\gamma}(t)=\boldsymbol{M}(\gamma(t))+c \text { and } \tilde{\boldsymbol{v}}_{i}(t)=\boldsymbol{M}\left(\boldsymbol{v}_{i}(t)\right), i=1,2
$$

for all $t \in I$, where the matrix $\boldsymbol{M}$ satisfies

$$
M^{\mathrm{T}} \boldsymbol{G} \boldsymbol{M}=\boldsymbol{G}, \quad \operatorname{det} \boldsymbol{M}=1, \quad \boldsymbol{G}=\left(\begin{array}{ccc}
-1 & 0 & 0 \\
0 & 1 & 0 \\
0 & 0 & 1
\end{array}\right)
$$


Proposition 1. For any vectors $\boldsymbol{a}$ and $\boldsymbol{b} \in \mathbb{R}_{1}^{3}$, we have

$$
\begin{aligned}
\langle\boldsymbol{a}, \boldsymbol{b}\rangle & =\langle\boldsymbol{M}(\boldsymbol{a}), \boldsymbol{M}(\boldsymbol{b})\rangle, \\
\boldsymbol{a} \wedge \boldsymbol{b} & =\boldsymbol{M}(\boldsymbol{a}) \wedge \boldsymbol{M}(\boldsymbol{b}) .
\end{aligned}
$$

The fundamental theorem of non-lightlike curves has been given in [20,21]. Referring to them and Proposition 1, we show the following theorem.

Theorem 2 (The Uniqueness Theorem). Let $\left(\gamma, v_{1}, v_{2}\right)$ and $\left(\tilde{\gamma}, \tilde{v}_{1}, \tilde{v}_{2}\right): I \rightarrow \mathbb{R}_{1}^{3} \times \Delta$ be spacelike framed curves whose curvatures $\left(\alpha(t), \ell_{1}(t), \ell_{2}(t), \ell_{3}(t)\right)$ and $\left(\tilde{\alpha}(t), \tilde{\ell}_{1}(t), \tilde{\ell}_{2}(t), \tilde{\ell}_{3}(t)\right)$ coincide. Then, $\left(\gamma, v_{1}, v_{2}\right)$ and $\left(\tilde{\gamma}, \tilde{v}_{1}, \tilde{v}_{2}\right)$ are congruent as spacelike framed curves through $a$ Lorentzian motion.

For a regular spacelike curve $\gamma: I \rightarrow \mathbb{R}_{1}^{3}$ with linear independent condition, that is, $\gamma^{\prime}(t)$ and $\gamma^{\prime \prime}(t)$ are linear independent for all $t \in I$. By direct calculations, we have the following proposition.

Proposition 2. Let $\left(\gamma, v_{1}, v_{2}\right): I \rightarrow \mathbb{R}_{1}^{3} \times \Delta$ be a spacelike framed curve with the curvature of the spacelike framed curve $\left(\alpha(t), \ell_{1}(t), \ell_{2}(t), \ell_{3}(t)\right)$. Then, the relationships between the $\left(\alpha(t), \ell_{1}(t), \ell_{2}(t), \ell_{3}(t)\right)$ and the curvature $\kappa(t)$ and torsion $\tau(t)$ of $\gamma$ are given by

$$
\begin{aligned}
& \kappa(t)=\frac{\sqrt{\left|\delta(t)\left(\ell_{3}^{2}(t)-\ell_{2}^{2}(t)\right)\right|}}{|\alpha(t)|}, \\
& \tau(t)=\frac{\ell_{2}^{\prime}(t) \ell_{3}(t)-\ell_{2}(t) \ell_{3}^{\prime}(t)+\ell_{1}(t) \ell_{2}^{2}(t)-\ell_{1}(t) \ell_{3}^{2}(t)}{\alpha(t)\left|\delta(t)\left(\ell_{3}^{2}(t)-\ell_{2}^{2}(t)\right)\right|} .
\end{aligned}
$$

\section{Nullcone Fronts of a Spacelike Framed Curve}

In this section, nullcone fronts of a spacelike framed curve are defined. Then, we give the classification theorem of their singularities as main theorem of the present paper.

Definition 2. For a spacelike framed curve $\left(\gamma, v_{1}, v_{2}\right): I \rightarrow \mathbb{R}_{1}^{3} \times \Delta$, we say

$$
\begin{aligned}
N F_{\gamma}^{+}: I \times \mathbb{R} & \longrightarrow \mathbb{R}_{1}^{3} \\
(t, u) & \longmapsto \gamma(t)+u\left(v_{1}(t)+v_{2}(t)\right)
\end{aligned}
$$

is the nullcone front of $\gamma$.

Remark 2. $N F_{\gamma}^{-}$be defined in the same way as above, which is also called the nullcone front of a spacelike framed curve.

Remark 3. We can verify that $v_{1}(t) \pm v_{2}(t)$ are null vectors. Therefore, the nullcone fronts $N F_{\gamma}^{ \pm}$ we defined above can be regarded as the surfaces formed by the trajectories of light rays emitted from each point on a spacelike curve $\gamma(t)$. This is a special relativity understanding of Fermat's principle that regards the light as particles. More detailed physical explanations can be found in [9].

The following theorem shows the singularity classification of the nullcone fronts we defined above.

Theorem 3. Let $\left(\gamma, v_{1}, v_{2}\right): I \rightarrow \mathbb{R}_{1}^{3} \times \Delta$ be a spacelike framed curve with $\ell_{2}(t) \pm \ell_{3}(t) \neq 0$, and then we have the following.

(1) The nullcone fronts $N F_{\gamma}^{ \pm}$of the curve $\gamma(t)$ are regular except the point $\left(t_{0}, u_{0}\right)$, where $u_{0}=-\frac{\alpha\left(t_{0}\right)}{\ell_{2}\left(t_{0}\right) \pm \ell_{3}\left(t_{0}\right)}$. 
(2) The nullcone fronts $N F_{\gamma}^{ \pm}$of the curve $\gamma(t)$ are diffeomorphic to the cuspidal edge $C \times \mathbb{R}$ at $\left(t_{0}, u_{0}\right)$ if and only if $\left(\alpha^{\prime}\left(t_{0}\right) \pm \alpha\left(t_{0}\right) \ell_{1}\left(t_{0}\right)\right)\left(\ell_{2}\left(t_{0}\right) \pm \ell_{3}\left(t_{0}\right)\right)-\alpha\left(t_{0}\right)\left(\ell_{2}^{\prime}\left(t_{0}\right) \pm \ell_{3}^{\prime}\left(t_{0}\right)\right) \neq 0$ and $u_{0}=-\frac{\alpha\left(t_{0}\right)}{\ell_{2}\left(t_{0}\right) \pm \ell_{3}\left(t_{0}\right)}$.

(3) The nullcone fronts $N F_{\gamma}^{ \pm}$of the curve $\gamma(t)$ are diffeomorphic to the swallowtail $S W$ at $\left(t_{0}, u_{0}\right)$ if and only if $\left(\alpha^{\prime}\left(t_{0}\right) \pm \alpha\left(t_{0}\right) \ell_{1}\left(t_{0}\right)\right)\left(\ell_{2}\left(t_{0}\right) \pm \ell_{3}\left(t_{0}\right)\right)-\alpha\left(t_{0}\right)\left(\ell_{2}^{\prime}\left(t_{0}\right) \pm \ell_{3}^{\prime}\left(t_{0}\right)\right)=$ $0,\left(\alpha^{\prime \prime}\left(t_{0}\right) \pm \alpha^{\prime}\left(t_{0}\right) \ell_{1}\left(t_{0}\right) \pm \alpha\left(t_{0}\right) \ell_{1}^{\prime}\left(t_{0}\right)\right)\left(\ell_{2}\left(t_{0}\right) \pm \ell_{3}\left(t_{0}\right)\right)^{3}-\left(\alpha\left(t_{0}\right) \ell_{2}^{\prime \prime}\left(t_{0}\right) \pm \alpha\left(t_{0}\right) \ell_{3}^{\prime \prime}\left(t_{0}\right)+\right.$ $\left.\alpha^{\prime}\left(t_{0}\right) \ell_{2}^{\prime}\left(t_{0}\right) \pm \alpha^{\prime}\left(t_{0}\right) \ell_{3}^{\prime}\left(t_{0}\right)\right)\left(\ell_{2}\left(t_{0}\right) \pm \ell_{3}\left(t_{0}\right)\right)^{2}+\alpha\left(t_{0}\right)\left(\ell_{2}^{\prime}\left(t_{0}\right) \pm \ell_{3}^{\prime}\left(t_{0}\right)\right)^{2}\left(\ell_{2}\left(t_{0}\right) \pm \ell_{3}\left(t_{0}\right)\right)$ $\neq 0$ and $u_{0}=-\frac{\alpha\left(t_{0}\right)}{\ell_{2}\left(t_{0}\right) \pm \ell_{3}\left(t_{0}\right)}$.

Here, $C \times \mathbb{R}=\left\{\left(x_{1}, x_{2}\right) \mid x_{1}^{2}=x_{2}^{3}\right\} \times \mathbb{R}$ is the cuspidal edge (Figure 1 ), and $S W=$ $\left\{\left(x_{1}, x_{2}, x_{3}\right) \mid x_{1}=v, x_{2}=4 u^{3}+2 u v, x_{3}=3 u^{4}+u^{2} v\right\}$ is the swallowtail (Figure 2 ).

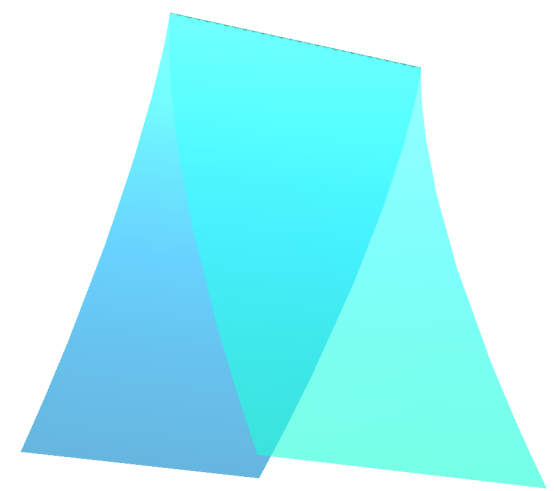

Figure 1. Cuspidal edge.

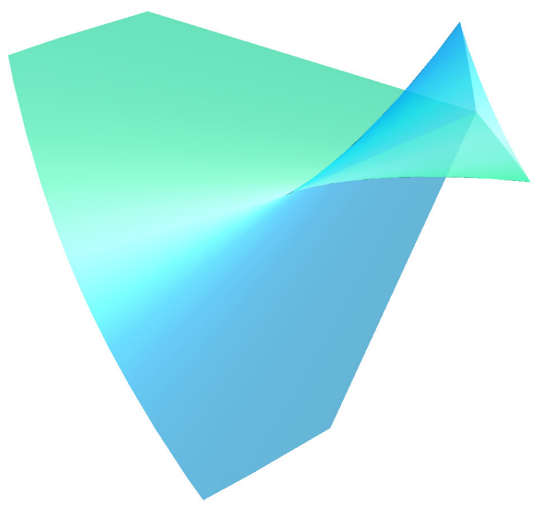

Figure 2. Swallowtail.

Remark 4. The regular spacelike curves and their nullcone fronts in Minkowski 3-space are discussed in the previous work of S. Izumiya, the second author and T. Sano [18]. In the present paper, the spacelike curve that we studied may contain singularities. Therefore, Theorem 3 is an extension of the conclusions (Theorem B) in [18].

For the sake of proving Theorem 3, we must make preparations in Section 4. Then, we will show the proof of Theorem 3 at the end of Section 4.

\section{Criteria for Singularities}

We review the criteria for singularities of fronts and related definitions briefly in this section. The word 'front' comes from physical fronts. Here, we use mathematical language to describe it. It is a generalization of the definition of a surface. More details can be found in $[22,23]$. In addition, we show the proof of Theorem 3 at the end of this section. 
We say a smooth map $f: U \subset \mathbb{R}^{2} \rightarrow \mathbb{R}_{1}^{3}$ is a front if there exists a vector field $v$ along $f$ such that $(f, v)$ is a Legendrian immersion. Then, there exists a smooth function $\lambda\left(u_{1}, u_{2}\right)$ on $U$, which is called the signed area density function, such that

$$
\frac{\partial f}{\partial u_{1}}\left(u_{1}, u_{2}\right) \wedge \frac{\partial f}{\partial u_{2}}\left(u_{1}, u_{2}\right)=\lambda\left(u_{1}, u_{2}\right) v\left(u_{1}, u_{2}\right)
$$

If a singular point $p \in U$ satisfies $\mathrm{d} \lambda(\boldsymbol{p}) \neq 0$, then $p$ is said to be non-degenerate. By parameterizing the set of singular points of $f$, we obtain the singular curve $\varphi(t)$, which satisfies $\varphi\left(t_{0}\right)=\boldsymbol{p}$. Then, we choose a null vector field $\eta(t)$ along $\varphi(t)$ such that $\mathrm{d} f(\eta(t))=0$ for each $t$.

Based on the definitions above, we show the following criteria for singularities. This not only simplifies the traditional proof process but also has a wider range of application.

Theorem 4 ([22,23]). Let $f: U \rightarrow \mathbb{R}_{1}^{3}$ be a front and $\boldsymbol{p} \in U$ be a non-degenerate singular point of $f$. Meanwhile, $\varphi(t)$ is the singular curve satisfies $\varphi\left(t_{0}\right)=\boldsymbol{p}$, and $\eta(t)$ is the corresponding null vector field. Then, we have the following.

(1) The germ of $f$ at $\boldsymbol{p}=\varphi\left(t_{0}\right)$ is locally diffeomorphic to a cuspidal edge if and only if the null direction $\eta\left(t_{0}\right)$ is transversal to $\varphi^{\prime}\left(t_{0}\right)$.

(2) The germ of $f$ at $\boldsymbol{p}=\varphi\left(t_{0}\right)$ is locally diffeomorphic to a swallowtail if and only if the null direction $\eta\left(t_{0}\right)$ is proportional to $\varphi^{\prime}\left(t_{0}\right)$ and

$$
\left.\frac{\mathrm{d}}{\mathrm{d} t}\right|_{t=t_{0}} \operatorname{det}\left(\varphi^{\prime}(t), \eta(t)\right) \neq 0
$$

Proof of Theorem 3. According to Definition 2 and formulae (1), we obtain

$$
\frac{\partial N F_{\gamma}^{ \pm}}{\partial t}(t, u) \wedge \frac{\partial N F_{\gamma}^{ \pm}}{\partial u}(t, u)=-\delta(t)\left(\alpha(t)+u\left(\ell_{2}(t) \pm \ell_{3}(t)\right)\right)\left(\boldsymbol{v}_{2}(t) \pm \boldsymbol{v}_{1}(t)\right)
$$

Thus, $\left(t_{0}, u_{0}\right)$ is a singular point of $N F_{\gamma}^{ \pm}(t, u)$ if and only if

$$
\alpha\left(t_{0}\right)+u_{0}\left(\ell_{2}\left(t_{0}\right) \pm \ell_{3}\left(t_{0}\right)\right)=0
$$

This assertion means that conclusion (1) in Theorem 3 holds.

Through the calculations above, we derive that $N F_{\gamma}^{ \pm}: I \times \mathbb{R} \rightarrow \mathbb{R}_{1}^{3}$ are fronts. Moreover, it can be verified that $\left(t_{0}, u_{0}\right)$ is a non-degenerate singular point of $N F_{\gamma}^{ \pm}(t, u)$ since $\ell_{2}\left(t_{0}\right) \pm \ell_{3}\left(t_{0}\right) \neq 0$.

We take the singular curve as

$$
\varphi(t)=\left(t,-\frac{\alpha(t)}{\ell_{2}(t) \pm \ell_{3}(t)}\right)
$$

and the null vector field as

$$
\eta(t)=\left(1, \pm \frac{\alpha(t) \ell_{1}(t)}{\ell_{2}(t) \pm \ell_{3}(t)}\right)
$$

Then, we obtain

$$
\begin{aligned}
\operatorname{det}\left(\varphi^{\prime}(t), \eta(t)\right) & =\frac{\left(\alpha^{\prime}(t) \pm \alpha(t) \ell_{1}(t)\right)\left(\ell_{2}(t) \pm \ell_{3}(t)\right)-\alpha(t)\left(\ell_{2}^{\prime}(t) \pm \ell_{3}^{\prime}(t)\right)}{\left(\ell_{2}(t) \pm \ell_{3}(t)\right)^{2}}, \\
\frac{\mathrm{d}}{\mathrm{d} t} \operatorname{det}\left(\varphi^{\prime}(t), \eta(t)\right) & =\frac{N(t)}{\left(\ell_{2}(t) \pm \ell_{3}(t)\right)^{4}},
\end{aligned}
$$

where $N(t)=\left(\alpha^{\prime \prime}(t) \pm \alpha^{\prime}(t) \ell_{1}(t) \pm \alpha(t) \ell_{1}^{\prime}(t)\right)\left(\ell_{2}(t) \pm \ell_{3}(t)\right)^{3}-\alpha(t)\left(\ell_{2}^{\prime \prime}(t) \pm \ell_{3}^{\prime \prime}(t)\right)\left(\ell_{2}(t) \pm\right.$ $\left.\ell_{3}(t)\right)^{2}+2 \alpha(t)\left(\ell_{2}^{\prime}(t) \pm \ell_{3}^{\prime}(t)\right)^{2}\left(\ell_{2}(t) \pm \ell_{3}(t)\right)-\left(2 \alpha^{\prime}(t) \pm \alpha(t) \ell_{1}(t)\right)\left(\ell_{2}^{\prime}(t) \pm \ell_{3}^{\prime}(t)\right)\left(\ell_{2}(t) \pm\right.$ 
$\left.\ell_{3}(t)\right)^{2}$. Then, by using the criteria for singularities (Theorem 4), conclusions (2) and (3) in Theorem 3 can be obtained.

Remark 5. According to the Equation (2) in the proof above, we know that if $\ell_{2}(t) \pm \ell_{3}(t)=0$, then the trajectory of the set of singularities $\left\{\left(t_{\alpha}, u\right) \mid \alpha\left(t_{\alpha}\right)=0, u \in \mathbb{R}\right\}$ of nullcone fronts $N F_{\gamma}^{ \pm}$ is a trivial regular curve.

\section{Examples}

In the following examples, we give specific expressions for different spacelike framed curves, whose original curves may contain singularities. We also show the expressions and figures of the nullcone fronts related to the spacelike framed curves. It can be seen that the nullcone fronts may have cuspidal edges and swallowtails.

Example 1. Let $\gamma_{1}(t)$ be a spacelike curve in $\mathbb{R}_{1}^{3}$ defined by

$$
\gamma_{1}(t)=\left(\frac{1}{2} t^{2}, \frac{1}{2} t^{2}, \frac{1}{4} t^{4}+\frac{1}{2} t^{2}\right)
$$

We can see that the origin is a singularity of the curve $\gamma_{1}(t)$. By defining

$$
\begin{aligned}
& v_{1}(t)=\frac{1}{\sqrt{\left(t^{2}+1\right)^{2}+1}}\left(0, t^{2}+1,-1\right), \\
& v_{2}(t)=\frac{1}{\sqrt{\left(t^{2}+1\right)^{2}\left(\left(t^{2}+1\right)^{2}+1\right)}}\left(\left(t^{2}+1\right)^{2}+1,1, t^{2}+1\right),
\end{aligned}
$$

we can say that $\left(\gamma_{1}, v_{1}, v_{2}\right): I \rightarrow \mathbb{R}_{1}^{3} \times \Delta$ is a spacelike framed curve.

As an example, we give the expression of $N F_{\gamma_{1}}^{ \pm}(t, u)$ as follows.

$$
\begin{aligned}
N F_{\gamma_{1}}^{ \pm}(t, u)= & \left(\frac{t^{2}}{2} \pm \frac{\left(\left(t^{2}+1\right)^{2}+1\right) u}{\sqrt{\left.\left(t^{2}+1\right)^{2}\left(t^{2}+1\right)^{2}+1\right)}},\right. \\
& \frac{t^{2}}{2}+\frac{\left(t^{2}+1\right) u}{\sqrt{\left(t^{2}+1\right)^{2}+1}} \pm \frac{u}{\sqrt{\left(t^{2}+1\right)^{2}\left(\left(t^{2}+1\right)^{2}+1\right)}} \\
& \left.\frac{t^{2}}{2}+\frac{t^{4}}{4}-\frac{u}{\sqrt{\left(t^{2}+1\right)^{2}+1}} \pm \frac{\left(t^{2}+1\right) u}{\sqrt{\left(t^{2}+1\right)^{2}\left(\left(t^{2}+1\right)^{2}+1\right)}}\right)
\end{aligned}
$$

we show the curve $\gamma_{1}(t)$ and nullcone front $N F_{\gamma_{1}}^{-}(t, u)$ of $\gamma_{1}(t)$ in Figure 3. We find that there exists a cuspidal edge on the nullcone front $N F_{\gamma_{1}}^{-}(t, u)$.

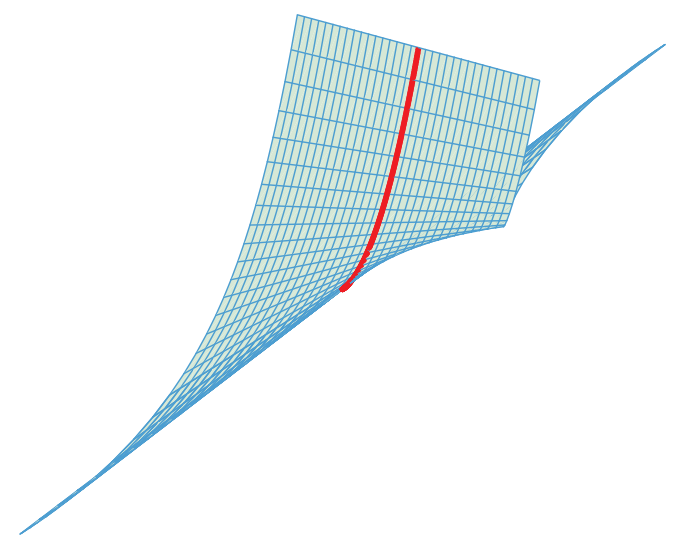

Figure 3. $\gamma_{1}(t)$ and $N F_{\gamma_{1}}^{-}(t, u)$. 
Example 2. Let $\gamma_{2}(t)$ be a spacelike curve in $\mathbb{R}_{1}^{3}$ defined by

$$
\gamma_{2}(t)=\left(\frac{t^{2}}{2}, \frac{t^{3}}{3}+\frac{t^{2}}{2}, \frac{t^{4}}{4}+\frac{t^{2}}{2}\right)
$$

which has a cusp (Figure 4). Then, $\left(\gamma_{2}, v_{1}, v_{2}\right): I \rightarrow \mathbb{R}_{1}^{3} \times \Delta$ is a spacelike framed curve, where

$$
\begin{aligned}
& v_{1}(t)=\frac{1}{\sqrt{\left(t^{2}+1\right)^{2}+(1+t)^{2}}}\left(0, t^{2}+1,-t-1\right), \\
& v_{2}(t)=\frac{1}{\sqrt{\left(t^{4}+3 t^{2}+2 t+1\right)\left(t^{4}+3 t^{2}+2 t+2\right)}}\left(\left(t^{2}+1\right)^{2}+(t+1)^{2}, t+1, t^{2}+1\right) .
\end{aligned}
$$

We show the curve $\gamma_{2}(t)$ and nullcone front

$$
\begin{aligned}
N F_{\gamma_{2}}^{+}(t, u)= & \left(\frac{t^{2}}{2}+\frac{\left(\left(t^{2}+1\right)^{2}+(t+1)^{2}\right) u}{\sqrt{\left(t^{4}+3 t^{2}+2 t+1\right)\left(t^{4}+3 t^{2}+2 t+2\right)}},\right. \\
& \frac{t^{3}}{3}+\frac{t^{2}}{2}+\frac{\left(t^{2}+1\right) u}{\sqrt{\left(t^{2}+1\right)^{2}+(t+1)^{2}}}+\frac{(t+1) u}{\sqrt{\left(t^{4}+3 t^{2}+2 t+1\right)\left(t^{4}+3 t^{2}+2 t+2\right)}} \\
& \left.\frac{t^{4}}{4}+\frac{t^{2}}{2}-\frac{(t+1) u}{\sqrt{\left(t^{2}+1\right)^{2}+(t+1)^{2}}}+\frac{\left(t^{2}+1\right) u}{\sqrt{\left(t^{4}+3 t^{2}+2 t+1\right)\left(t^{4}+3 t^{2}+2 t+2\right)}}\right)
\end{aligned}
$$

of $\gamma_{2}(t)$ in Figure 5. We find that the nullcone front $\mathrm{NF}_{\gamma_{2}}^{+}(t, u)$ of $\gamma_{2}(t)$ has a cuspidal edge.

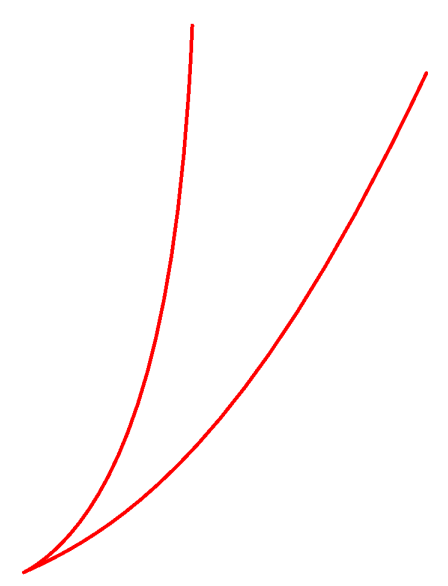

Figure 4. $\gamma_{2}(t)$.

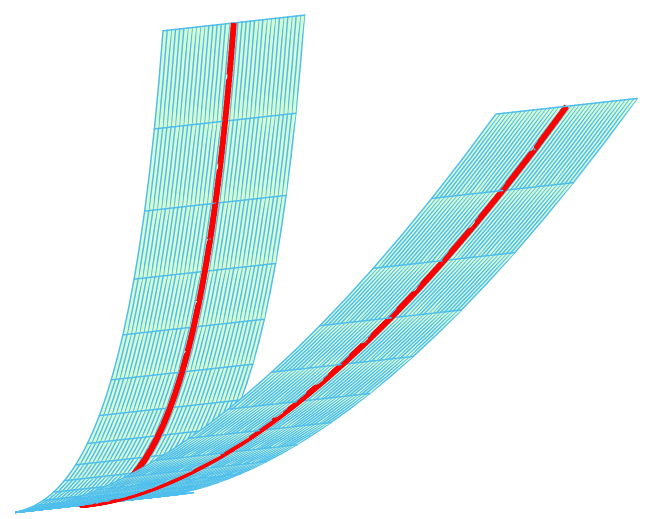

Figure 5. $\gamma_{2}(t)$ and $N F_{\gamma_{2}}^{+}(t, u)$. 
Example 3. Let $\gamma_{3}(t)$ be a spacelike curve in $\mathbb{R}_{1}^{3}$ defined by

$$
\gamma_{3}(t)=(0,2 \cos t, \sin t)
$$

If we take

$$
\begin{aligned}
& v_{1}(t)=\frac{1}{\sqrt{3 \sin ^{2} t}}(1, \cos t, 2 \sin t) \\
& v_{2}(t)=\frac{1}{\sqrt{3 \sin ^{2} t\left(3 \sin ^{2} t+1\right)}}\left(3 \sin ^{2} t+1, \cos t, 2 \sin t\right),
\end{aligned}
$$

then $\left(\gamma_{3}, v_{1}, v_{2}\right): I \rightarrow \mathbb{R}_{1}^{3} \times \Delta$ is a spacelike framed curve. We show the curve $\gamma_{3}(t)$ and nullcone front

$$
\begin{aligned}
N F_{\gamma_{3}}^{+}(t, u)= & \left(\frac{u\left(3 \sin ^{2} t+1\right)}{\sqrt{3 \sin ^{2} t\left(3 \sin ^{2} t+1\right)}}+\frac{u}{\sqrt{3 \sin ^{2} t}},\right. \\
& 2 \cos t+\frac{u \cos t}{\sqrt{3 \sin ^{2} t\left(3 \sin ^{2} t+1\right)}}+\frac{u \cos t}{\sqrt{3 \sin ^{2} t}}, \\
& \left.\sin t+\frac{2 u \sin t}{\sqrt{3 \sin ^{2} t\left(3 \sin ^{2} t+1\right)}}+\frac{2 u \sin t}{\sqrt{3 \sin ^{2} t}}\right)
\end{aligned}
$$

of $\gamma_{3}(t)$ in Figure 6. We can see that the nullcone front $N F_{\gamma_{3}}^{+}(t, u)$ of $\gamma_{3}(t)$ has swallowtails.

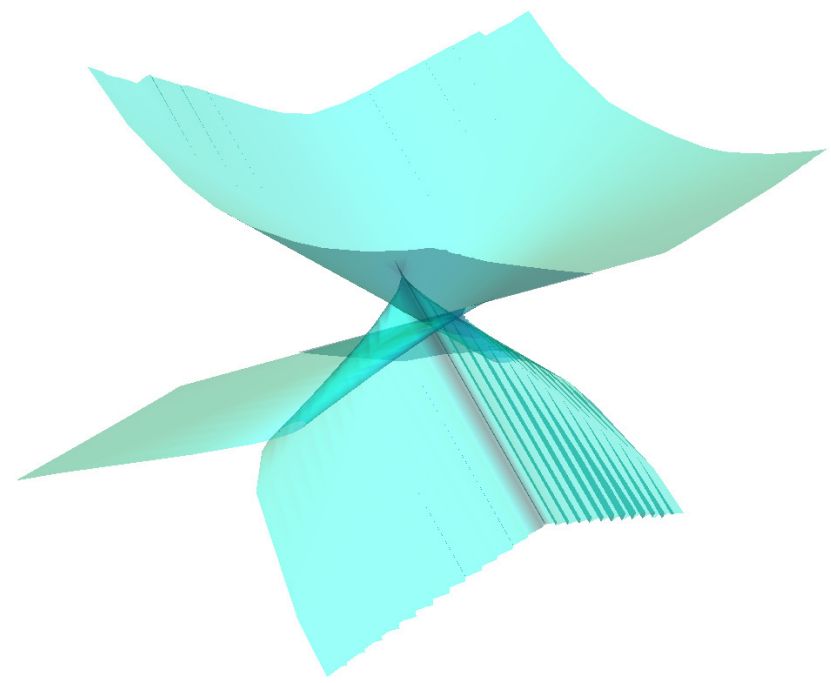

Figure 6. $N F_{\gamma_{3}}^{+}(t, u)$.

Remark 6. The original curve in Example 3 is a regular spacelike curve. This example shows that the spacelike framed curve is still valid for the regular spacelike curve-that is, it is an extension of Frenet curves. We can use it to study both regular spacelike curves and singular spacelike curves in Minkowski 3-space.

\section{Conclusions}

In this paper, we presented the definition of spacelike framed curves in Minkowski 3-space. This is a new useful tool to study spacelike curves in Minkowski 3-space, even if they are singular. Furthermore, we provided notions of nullcone fronts with respect to a spacelike framed curve, and these fronts are formed by light rays emitted from the spacelike curve. For the purpose of analyzing the singular properties, we introduced the concept of a front and the criterion for singularities, which is applicable to a wider range 
and easy to calculate. Then, we analyzed their singular properties using the curvature of the spacelike framed curve.

Author Contributions: Writing-Original Draft Preparation, Writing-Review and Editing, P.L.; Conceptualization, Methodology, Funding Acquisition, D.P. All authors have read and agreed to the published version of the manuscript.

Funding: This research was funded by National Natural Science Foundation of China (Grant No. 11671070).

Institutional Review Board Statement: Not applicable.

Informed Consent Statement: Not applicable.

Data Availability Statement: Not applicable.

Acknowledgments: The authors would like to thank the editors and referees for helpful comments to improve the original paper. Moreover, we would like to express our gratitude to the referees who provided detailed suggestions for our further research.

Conflicts of Interest: The authors declare no conflict of interest.

\section{References}

1. O'Neill, B. Semi-Riemannian Geometry; Academic Press: New York, NY, USA, 1983.

2. Duggal, K.L.; Jin, D.H. Null Curves and Hypersurfaces of Semi-Riemannian Manifolds; World Scientific: Singapore, 2007.

3. Li, Y.; Wang, Z. Lightlike tangent developables in de Sitter 3-space. [CrossRef] J. Geom. Phys. 2021, 164, 104188. [CrossRef]

4. Sun, J. Singularity properties of null Killing magnetic curves in Minkowski 3-space. Int. J. Geom. Methods Mod. Phys. 2020, 17, 2050141. [CrossRef]

5. Sun, J.; Jiang, X.; Ji, F. Geometrical Properties of the Pseudonull Hypersurfaces in Semi-Euclidean 4-Space. Mathematics 2021, 9, 1274. [CrossRef]

6. Wei, S.; Huang, J.; Chen, L. Singularities of null surfaces of null Cartan curves in three-dimensional anti-de Sitter space. Topol. Appl. 2018, 234, 238-247. [CrossRef]

7. Ateş, F.; Ekmekci, F. Light patterns generated by the reflected rays. Optik 2020, 224, 165507. [CrossRef]

8. Ateş, F. Singularities of reflected spherical light rays from spacelike curve on the de Sitter 3-space. Optik 2021, $242,167303$.

9. Izumiya, S. Wave Front Propagations and Singularities; Kyoritsu Publishing Co., Ltd.: Tokyo, Japan, 2018. (In Japanese)

10. O'Neill, B. Elementary Differential Geometry, Revised 2nd ed.; Academic Press: New York, NY, USA, 2006.

11. Bishop, R. There is more than one way to frame a curve. Amer. Math. Mon. 1975, 82, 246-251.

12. Li, P.; Pei, D. Evolutes and focal surfaces of $(1, k)$-type curves with respect to Bishop frame in Euclidean 3-space. Math. Meth. Appl. Sci. 2021. [CrossRef]

13. Y1lmaz, S.; Turgut, M. A new version of Bishop frame and an application to spherical images. J. Math. Anal. Appl. 2010, 371, 764-776. [CrossRef]

14. Honda, S.; Takahashi, M. Framed curves in the Euclidean space. Adv. Geom. 2016, 16, 265-276. [CrossRef]

15. Li, Y.; Pei, D.; Takahashi, M.; Yu, H. Envelopes of Legendre curves in the unit spherical bundle over the unit sphere. Q. J. Math. 2018, 69, 631-653. [CrossRef]

16. Wang, Y.; Pei, D.; Gao, R. Generic Properties of Framed Rectifying Curves. Mathematics 2019, 7, 37. [CrossRef]

17. Yazıcı, B.; Karakuş, S.; Tosun, M. On the classification of framed rectifying curves in Euclidean space. Math. Meth. Appl. Sci. 2021. [CrossRef]

18. Izumiya, S.; Pei, D.; Sano, T. The lightcone Gauss map and the lightcone developable of a spacelike curve in Minkowski 3-space. Glasg. Math. J. 2000, 42, 75-89. [CrossRef]

19. Walrave, J. Curves and Surfaces in Minkowski Space. Ph.D. Thesis, Katholieke Universiteit Leuven, Leuven, Belgium, 1995.

20. Honda, A. Behavior of torsion functions of spacelike curves in Lorentz-Minkowski space. arXiv 2019, arXiv: 1905.03367. Available online: https:/ / arxiv.org/pdf/1905.03367.pdf (accessed on 19 September 2021).

21. Lopez, R. Differential geometry of curves and surfaces in Lorentz-Minkowski space. Int. Electron. J. Geom. 2014, 7, 44-107. [CrossRef]

22. Kokubu, M.; Rossman, W.; Saji, K.; Umehara, M.; Yamada, K. Singularities of flat fronts in hyperbolic space. Pac. J. Math. 2005, 221, 303-351. [CrossRef]

23. Saji, K.; Umehara, M.; Yamada, K. The geometry of fronts. Ann. Math. 2009, 169, 491-529. [CrossRef] 